\title{
Image Fusion With No Gamut Problem by Improved Nonlinear IHS Transforms for Remote Sensing
}

\author{
Chun-Liang Chien and Wen-Hsiang Tsai, Senior Member, IEEE
}

\begin{abstract}
An image fusion method must ideally preserve both the detail of the panchromatic image and the color of the multispectral image. Existing image fusion methods incur the gamut problem of creating new colors which fall out of the RGB cube. These methods solve the problem by color clipping which yields undesirable color distortions and contrast reductions. An improved nonlinear IHS (intensity, hue, saturation; iNIHS) color space and related color transformations are proposed in this paper to solve the gamut problem without appealing to color clipping. The iNIHS space includes two halves, one being constructed from the lower half of the RGB cube by RGB to IHS transformations, and the other from the upper half of the RGB cube by CMY to IHS transformations. While incurring no out-of-gamut colors, desired intensity substitutions and additions in substitutive and additive image fusions, respectively, are all achievable, with the saturation component regulated within the maximum attainable range. Good experimental results show the feasibility of the proposed method.
\end{abstract}

Index Terms-Clipping, gamut problem, image fusion, improved nonlinear IHS (iNIHS) color space, multispectral image, panchromatic image.

\section{INTRODUCTION}

D IFFERENT spaceborne sensors yield remotely sensed images with different characteristics, like panchromatic (PAN) images which are black and white with higher resolutions as well as multispectral (MS) images which are colorful with lower resolutions. It is advantageous to combine a PAN image and an MS image to create a third image without the weaknesses of the two original ones, i.e., a color image with a higher resolution. An ideal image fusion method for this purpose must preserve simultaneously the detail of the former and the color of the latter. The resulting pan-sharpened image is useful for applications like feature detection, land cover classification, visual inspection, etc. [1]-[3]. For example, for map browsing using tools like the Google Earth, it is desired to generate maps with resolutions as high as possible by com-

Manuscript received June 18, 2012; revised October 31, 2012; accepted December 22, 2012. Date of publication March 13, 2013; date of current version November 26, 2013. This work was supported financially by the Ministry of Education, Taiwan and National Chiao Tung University, Taiwan under the five-year Project of "Aiming for the Top University" from 2011-2015.

C.-L. Chien is with the Department of Biomedical Engineering, Chun Yuan Christian University, Taoyuan 32023, Taiwan (e-mail: jameschyan@cycu. edu.tw).

W.-H. Tsai is with the Department of Computer Science, National Chiao Tung University, Hsinchu 30010, Taiwan, and also with the Department of Information Communication, Asia University, Taichung 41354, Taiwan (e-mail: whtsai@cis.nctu.edu.tw).

Color versions of one or more of the figures in this paper are available online at http://ieeexplore.ieee.org.

Digital Object Identifier 10.1109/TGRS.2013.2243157 bining earth images acquired from multiple sources, and this is exactly the issue dealt with in this study.

Many techniques have been proposed for image fusion [4], [5], e.g., the IHS (intensity, hue, saturation) transformation [6]-[10], principal component analysis (PCA) [3], independent component analysis (ICA) [11], arithmetic combination [4], [12], the least-square support vector machine [13], the wavelet transformation [3], [14]-[18], the contourlet transformation [19], and the generalized Laplacian pyramid with context-based decisions (GLP-CBD) [15]. In these techniques, the GLP-CBD has been recognized superior to many others according to [20]. However, these methods do not touch the out-of-gamut problem, or simply, the gamut problem, which arises when a transformed color value falls outside the range of the involved color space, except Schetselaar [6] who discussed the difference between the effects yielded by the spherical and cylindrical transformations in image fusion: when the intensity value is changed excessively, the former transformation might cause over-saturation in the higher intensity range while the latter might do so in both the higher and lower intensity ranges.

To solve the gamut problem, a solution is to clip each outof-gamut color to fit it back into the RGB range, called the $R G B$ cube. For this, Yang and Kwok [21] presented three clipping techniques and revealed the facts: 1) clipping a pixel's RGB values in most cases will change its intensity, hue, and saturation values, as well as its color if the processed color is far away from the RGB cube; 2) clipping the saturation value will improve the contrast because each pixel can reach the expected intensity, but the spectral energy in saturation is lost, causing some details to disappear; 3 ) clipping the intensity value will not change the chromatic attribute, but will degrade the contrast and blur image details. Similar situations have been mentioned in [7], [21], and [22]. It is aimed to solve the gamut problem without using the clipping operation in this study.

To see the gamut problem in detail, a common technique [22], [23] of transforming the intensity and saturation values in an MS image $M S$ while keeping the hue values unaltered is elaborated here. Two operations in the RGB space, scaling and shifting, were introduced to express the effect of such a type of color transformation as described in the following:

$$
\left[r^{\prime}, g^{\prime}, b^{\prime}\right]^{T}=\gamma \times[r, g, b]^{T}+[\delta, \delta, \delta]^{T}
$$

where $[r, g, b]^{T}$ is the color of a pixel $p$ in $M S ;\left[r^{\prime}, g^{\prime}, b^{\prime}\right]^{T}$ is the color of the corresponding pixel $p^{\prime}$ in the resulting image; and $\gamma$ and $\delta$ are two factors used for scaling and shifting, respectively, with $T$ denoting the "vector transpose" operation. When $\gamma=$ 1 , the transformation becomes a shifting operation, and when 
$\delta=0$, a scaling one. Let the PAN image used in the fusion be denoted as $P A N$ and the pixel in $P A N$ corresponding to $p$ as $p^{\prime \prime}$. Following Tu et al. [7], four types of techniques may be identified from the existing methods in terms of (1) with their factors $\gamma$ and $\delta$ defined as follows.

1) Fusion using the linear IHS transformation [6]-[9]. $\gamma=$ 1 and $\delta=P A N\left(p^{\prime \prime}\right)-I(p)$ where $I(p)$ is the intensity value of pixel $p$ in image $M S$ computed as $I(p)=(r+$ $g+b) / 3$; and $P A N\left(p^{\prime \prime}\right)$ is the value of pixel $p^{\prime \prime}$ in image $P A N$.

2) Fusion using PCA or ICA techniques [3], [11]. $\gamma=1$ and $\delta=P A N\left(p^{\prime \prime}\right)-P C^{1}(p)$ where $P C^{1}(p)$ is the value of pixel $p$ in the first principal or independent component of $M S$.

3) Fusion using the wavelet transform [3], [14]-[18]. $\gamma=$ 1 and $\delta=P A N\left(p^{\prime \prime}\right)-P A N_{\mathrm{r}}(p)$ where $P A N_{\mathrm{r}}(p)$ is a smooth version of image $P A N$ obtained from a wavelet transform of $P A N$.

4) Fusion using the Brovey transformation [4], [12]. $\gamma=$ $P A N\left(p^{\prime \prime}\right) / I(p)$ and $\delta=0$.

Each of the above methods uses just a shifting or scaling operation, but not both, for image fusion. Recently, Choi [24] proposed the use of a tradeoff parameter $l$ to control the degrees of preserved spatial information and spectral distortion in the resulting image, which is essentially a shifting operation. Later, Tu et al. [25] improved the method by using both shifting and scaling operations to achieve a better tradeoff. These two methods may be described in terms of (1) in the following.

5) Fusion using the Choi method [24]. $\gamma=1$ and $\delta=[(l-$ 1) $/ l] \times\left[P A N\left(p^{\prime \prime}\right)-I(p)\right]$.

6) Fusion usingthe method proposed by Tu et al. [25]. $\gamma=$ $P A N\left(p^{\prime \prime}\right) /\left\{[(l-1) / l] P A N\left(p^{\prime \prime}\right)+(1 / l) I(p)\right\}$ and $\delta=$ $\gamma \times[(l-1) / l] \times\left[P A N\left(p^{\prime \prime}\right)-I(p)\right]$.

The above methods do not guarantee the resulting color to be within the RGB cube; and if it is so, i.e., if it is out of the gamut, a clipping technique is performed, as mentioned before.

In short, no matter which clipping method is used, in general, no good fusion result can be produced. To overcome this problem, we propose in this paper an improved nonlinear IHS (iNIHS) color space for use in image fusion. The gamut problem can so be avoided and clipping becomes unnecessary. When the intensity component is processed in the proposed iNIHS color space, the saturation component can be adjusted within the maximum attainable saturation range, so that all the desired intensity changes can be achieved with the contrast being well kept. Moreover, all the saturation values of the pixels at the same intensity level are proportionally expanded or shrunk in such a way that the spectral energy of saturation is preserved.

In the remainder of this paper, the gamut problem incurred by linear and nonlinear IHS color transformations is introduced in Section II where intensity substitution and color clipping techniques are also reviewed. In Section III, the proposed iNIHS space and related transformations are described. In Section IV, experimental results of substitutive and additive image fusions are presented, and conclusions are drawn finally in Section V.

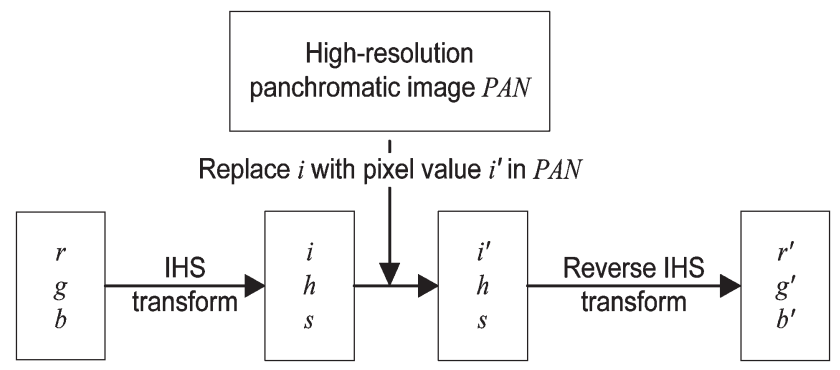

Fig. 1. Illustration of IHS transformation for image fusion.

\section{IMAGE FUSIONS IN LINEAR AND NONLINEAR IHS SPACES}

The RGB to IHS color transformation, or simply the IHS transformation, is often applied in image fusion [6]-[9], [24][27] because the intensity, hue, and saturation components can be perceived more intuitively, and manipulated separately. Image fusion via the IHS color space, as illustrated in Fig. 1, consists of the following three steps:

1) transform the RGB values $(r, g, b)$ of each pixel in the input MS image $M S$ into the IHS space to get the corresponding IHS values $(i, h, s)$;

2) replace the obtained intensity component $i$ with the corresponding pixel's intensity value $i^{\prime}$ in the input PAN image $P A N$; and

3) regard $\left(i^{\prime}, h, s\right)$ as new IHS values and transform them back into the RGB space to obtain new RGB values $\left(r^{\prime}, g^{\prime}, b^{\prime}\right)$.

The above process is called substitutive image fusion [17], which may be compared with additive image fusion to be introduced later in Section IV. The ways of intensity substitutions (i.e., replacing $i$ with $i^{\prime}$ ) in the linear and nonlinear IHS transformations adopted in the existing methods are different: the linear transformation uses shifting and the nonlinear one scaling. Such intensity scaling and shifting operations will be reviewed in Section II-A, and the incurred gamut problem described in Section II-B.

\section{A. Intensity Substitutions in IHS Transformations}

The linear IHS transformation and its reverse [7] may described, respectively, as follows.

\section{Linear RGB to IHS and IHS to RGB transformations}

$$
\begin{aligned}
{[i, h, s]^{T}=} & \boldsymbol{A} \times[r, g, b]^{T}, \\
& \text { with } \boldsymbol{A}=\left[\begin{array}{ccc}
1 / 3 & 1 / 3 & 1 / 3 \\
-\sqrt{2} / 6 & -\sqrt{2} / 6 & 2 \sqrt{2} / 6 \\
1 / \sqrt{2} & -1 / \sqrt{2} & 0
\end{array}\right] \\
{\left[r^{\prime}, g^{\prime}, b^{\prime}\right]^{T}=} & \boldsymbol{B} \times\left[i^{\prime}, h^{\prime}, s^{\prime}\right]^{T}, \\
& \text { with } \boldsymbol{B}=\left[\begin{array}{ccc}
1 & -1 / \sqrt{2} & 1 / \sqrt{2} \\
1 & -1 / \sqrt{2} & -1 / \sqrt{2} \\
1 & \sqrt{2} & 0
\end{array}\right] .
\end{aligned}
$$

By the above transformations, the effect of conducting intensity substitution $i^{\prime}=i+\delta$ in the IHS space may be shown to be just a direct color shifting in the original RGB space as follows:

$$
\begin{aligned}
{\left[r^{\prime}, g^{\prime}, b^{\prime}\right]^{T} } & =\boldsymbol{B} \times\left[i^{\prime}, h, s\right]^{T}=\boldsymbol{B} \times[i+\delta, h, s]^{T} \\
& =[r, g, b]^{T}+[\delta, \delta, \delta]^{T} .
\end{aligned}
$$


On the other hand, the nonlinear IHS color transformation, called the HSI color transformation in the image processing domain [28], is defined as follows.

\section{Nonlinear RGB to IHS transformation}

$a=\frac{(2 b-g-r) / 2}{\sqrt{(b-g)^{2}+(b-r)(g-r)}} ; \quad i=\frac{r+g+b}{3} ;$

$h=\left\{\begin{array}{ll}\cos ^{-1}(a) & \text { if } b \leq g ; \\ 360^{\circ}-\cos ^{-1}(a) & \text { if } b>g ;\end{array}, s=1-\frac{3 \times \min (r, g, b)}{r+g+b}\right.$

where $h \in\left[0^{\circ}, 360^{\circ}\right]$, and $i, s \in[0,1]$. The reverse transformation may be described as follows.

\section{Nonlinear IHS to RGB transformation}

1) Section RG $\left(0^{\circ} \leq h<120^{\circ}\right)$

$$
\begin{aligned}
& b=i(1-s) ; \quad r=i\left[1+s \cos h / \cos \left(60^{\circ}-h\right)\right] ; \\
& g=3 i-(r+b) .
\end{aligned}
$$

2) Section GB $\left(120^{\circ} \leq h<240^{\circ}\right)$

$$
\begin{aligned}
& h=h-120^{\circ} ; \quad r=i(1-s) ; \\
& g=i\left[1+s \cos h / \cos \left(60^{\circ}-h\right)\right] ; b=3 i-(r+g) .
\end{aligned}
$$

3) Section $\operatorname{BR}\left(240^{\circ} \leq h<360^{\circ}\right)$

$$
\begin{aligned}
h & =h-240^{\circ} ; \quad g=i(1-s) ; \\
b & =i\left[1+s \cos h / \cos \left(60^{\circ}-h\right)\right] ; \quad r=3 i-(g+b) .
\end{aligned}
$$

By using the above transformations, for section GB, e.g., the effect of conducting the intensity substitution $i^{\prime}=\gamma \times i$ in the nonlinear IHS color space may be shown to be just a direct color scaling in the original RGB space as follows:

$$
\left[\begin{array}{l}
r^{\prime} \\
g^{\prime} \\
b^{\prime}
\end{array}\right]=\left[\begin{array}{l}
i^{\prime}(1-s) \\
i^{\prime}\left[1+s \cos h / \cos \left(60^{\circ}-h\right)\right] \\
3 i^{\prime}-\left(r^{\prime}+g^{\prime}\right)
\end{array}\right]=\frac{i^{\prime}}{i}\left[\begin{array}{l}
r \\
g \\
b
\end{array}\right]=\gamma\left[\begin{array}{l}
r \\
g \\
b
\end{array}\right] .
$$

For sections RG and BR, similar conclusions may be drawn using (5), (6), and (8).

As a summary, combining (4) and (9) leads to a general equation for image fusion described by (1). In particular, if the intensity $i$ is replaced by $i^{\prime}=i+\delta$ in the linear IHS space, the effect is to shift the RGB values by the amount $\delta$ so that $\left[r^{\prime}, g^{\prime}, b^{\prime}\right]^{T}=[r, g, b]^{T}+[\delta, \delta, \delta]^{T}$. And if the intensity $i$ is substituted by $i^{\prime}=\gamma \times i$ in the nonlinear IHS space, the effect is to rescale the RGB values by the factor $\gamma$ so that $\left[r^{\prime}, g^{\prime}, b^{\prime}\right]^{T}=\gamma \times[r, g, b]^{T}$.

\section{B. Gamut Problem in Linear and Nonlinear IHS Color Spaces}

Color images processed in spaces other than the RGB one usually are transformed back into the RGB space for displays. If a pixel's color is back-projected to fall outside the RGB cube, a gamut problem is said to occur. Both scaling and shifting operations may incur the gamut problem [6]. Solving such problems using clipping will lead to contrast reductions and color distortions, as mentioned previously.

It is tried in this study to solve the gamut problem without using clipping. For this, 3-D color models are created to observe the problem intuitively. Specifically, each face of the RGB cube is divided into $21 \times 21$ patches, and the values of the

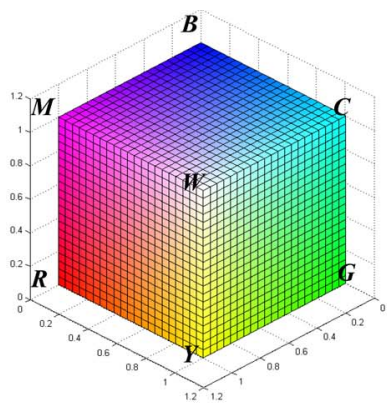

(a)

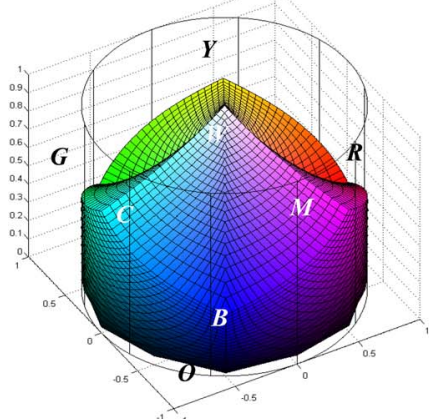

(c)

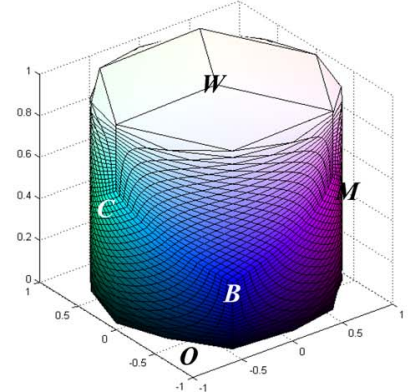

(e)

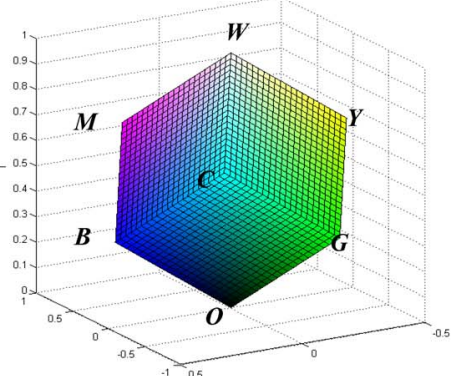

(b)

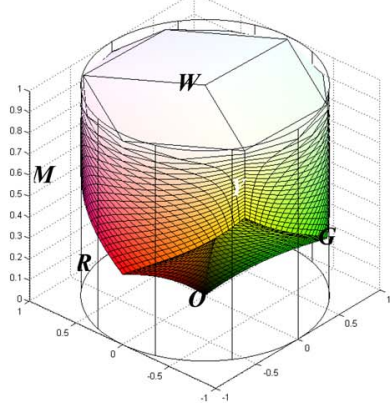

(d)

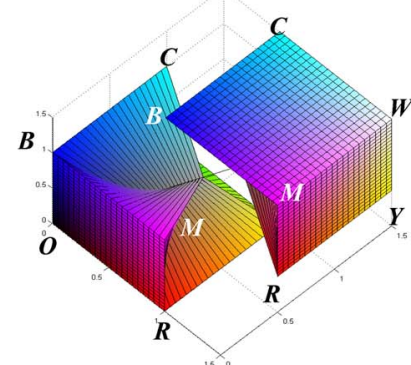

(f)

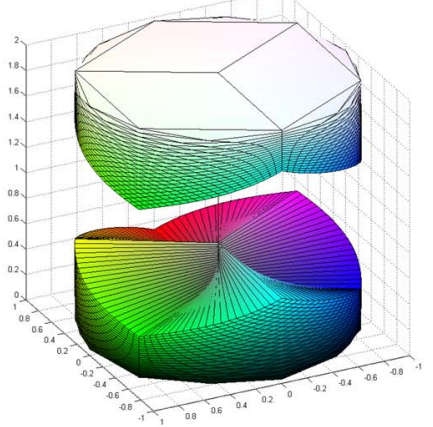

(g)

Fig. 2. Color model transformations. (a) RGB cube. (b) Linear IHS model based on RGB color. (c) Nonlinear IHS model based on RGB color. (d) Nonlinear IHS model based on CMY color. (e) Proposed iNIHS color model resulting from merging (c) and (d). (f) RGB cube divided into two parts by proposed method. (g) Transformation of (e) into iNIHS space.

color points on each patch are computed by inverse distance weighted interpolation in terms of the colors of the four vertices of the patch. The RGB color model so generated is shown in Fig. 2(a). Then, the model is transformed into other color spaces to yield new models for observations. The linear and nonlinear IHS color models so obtained are shown in Fig. 2(b) and (c), respectively. 
From Fig. 2(b), it can be seen that if the intensity of a color point within the resulting IHS cube in the figure is replaced with one outside the cube during image fusion, then after the backward linear IHS transformation is conducted, the resulting RGB color point will no longer be within the RGB cube shown in Fig. 2(a), causing a gamut problem. In more detail, it is noted that the upper portion of the resulting nonlinear IHS model as shown in Fig. 2(c) is "collapsed," meaning that the top of the model, supposedly a flat "circular-shaped region" with the point $W$ as the center, becomes a lower "roof with a waveshaped surface," where the wave is composed of the six corner points of $B, C, G, Y, R$, and $M$ around the roof boundary. This phenomenon comes from the fact that the saturation values of those non-boundary points on the roof are not normalized to the maximum extent of the value 1 in the transformation process. Consequently, if manipulations on the intensity values in the image fusion process yield an IHS color point $P^{\prime}$ falling into the collapsed portion between the "imagined" flat circularshaped top and the wave-shaped roof, then a gamut problem will occur because a backward nonlinear IHS transformation will obviously convert $P^{\prime}$ to be an RGB color point falling outside the RGB cube shown in Fig. 2(a).

\section{Proposed Improved INIHS SPACE}

To solve the previously described gamut problem in the backward nonlinear IHS to RGB transformation, the collapsed portion mentioned above should be "repaired" to yield a shape of a complete circle on top of the cylinder as shown in Fig. 2(e) instead of a wave-shaped collapsed roof at a lower height. That is, a complete cylindrical IHS color space with a circular-shaped top should be created to include any color point resulting from image fusion; and such a new IHS color space, when transformed nonlinearly back into the RGB space, should fit exactly into the RGB cube to cause no gamut problem. To implement this idea to create the improved nonlinear IHS (iNIHS) color space, the proposed method is described next.

First, as can be seen from Fig. 2(a), the RGB space with the black-color vertex as its origin, which is denoted as $O$ in Fig. 2(b) and (c) but not seen in Fig. 2(a), may be considered alternatively as a CMY color space with the white-color vertex as its origin, which is denoted as $W$ in the figures. Secondly, if we transform not only the RGB space but also the CMY space into the nonlinear IHS color space, we will get respectively two transformation results, with one already shown in Fig. 2(c) and the other shown newly in Fig. 2(d). It can be seen that Fig. 2(d) is just an upside-down version of Fig. 2(c). Finally, if we merge the two results together, we will get an extended IHS space as shown in Fig. 2(e) which is close to the shape of a cylinder with a non-flat circular-shaped top. If the sampling grid size is taken to be finer and finer, then the top of the cylinder will eventually become a flat circular shape. This extended cylindrical-shaped IHS space is just the previously mentioned iNIHS color space proposed in this paper for use in image fusion. In this way, it is seen that if a color point obtained from image fusion falls into the aforementioned collapsed portion in Fig. 2(c), we may now transform the color point, using a backward IHS to $C M Y$ transformation instead of a backward IHS to $R G B$ one, into a CMY color point which appears definitely within the original RGB cube!

In more detail, after image fusion we may simply transform the upper half of the iNIHS space as shown in Fig. 2(d) into the CMY space via a backward IHS to $C M Y$ transformation (not via a backward IHS to $R G B$ transformation), and transform the lower half of the cylinder-shaped IHS space as shown in Fig. 2(c) into the RGB space via a backward IHS to $R G B$ transformation as done conventionally. To do this, some way to cut the iNIHS color space into two halves should be found out, or equivalently, a boundary surface between the two halves should be defined. This boundary surface, denoted as $B S_{\text {iNIHS }}$, in the iNIHS color space, when transformed back into the united $R G B-C M Y$ color space, will result in a surface, denoted as $B S_{\mathrm{RGB}}$, which cuts the original RGB cube shown in Fig. 2(a) into two symmetric halves as shown in Fig. 2(f), with one taken as part of the RGB space and the other as part of the CMY space. These two halves will be denoted as $H_{\mathrm{RGB}}$ and $H_{\mathrm{CMY}}$, respectively, hereafter. Reversely, about the forward transformation from the united RGB-CMY space, we may map $H_{\mathrm{CMY}}$ into the upper half of the iNIHS space above the boundary surface $B S_{\mathrm{iNIHS}}$, and $H_{\mathrm{RGB}}$ into the lower half of the iNIHS space below $B S_{\mathrm{iNIHS}}$, as shown in Fig. 2(g).

The issue now is how to specify the boundary surfaces $B S_{\mathrm{RGB}}$ and $B S_{\mathrm{iNIHS}}$ in the RGB and iNIHS spaces, respectively. The way we propose for this aim is described next in Section III-A. The corresponding RGB to iNIHS transformation will be derived in Section III-B, and the corresponding iNIHS to RGB transformation in Section III-C. Differences between the proposed iNIHS color space and the conventional nonlinear IHS space will be described in Section III-D. Finally, the proposed method for solving the gamut problem will described in detail in Section III-E.

\section{A. Derivations of the Boundary Surfaces Between the Halves of RGB and iNIHS Spaces}

To derive equations to specify the boundary surface $B S_{\text {iNIHS }}$ in the iNIHS color space, we define several terms at first. Referring to Fig. 3(a), we define the gray axis of the RGB cube as the diagonal line $\overline{O W}$ going through the two color corners, $O$ and $W$, of the cube. Note that when the RGB cube is used in the linear IHS color model as shown in Fig. 2(b), the cube is re-oriented so that this gray axis becomes perpendicular to the horizontal zero-valued intensity plane. Also, we define the horizontal saturation line $L_{c}$ for a color point $c$ as the line going through $c$ and perpendicular to gray axis $\overline{O W}$. Let the intersection point of $L_{c}$ with $\overline{O W}$ be denoted as $c^{\prime}$ and that of $L_{c}$ with the RGB cube surface as $c^{\prime \prime}$. Then, we define the maximum attainable saturation range $R_{c}$ of color point $c$ as the length of line segment $\overline{c^{\prime} c^{\prime \prime}}$. Furthermore, the boundary surface $B S_{\mathrm{RGB}}$ between the upper and lower halves, $H_{\mathrm{RGB}}$ and $H_{\mathrm{CMY}}$, as depicted in Fig. 2(f), is redrawn in Fig. 3(b) and (c) from two different views, which is composed of six patches connected in a wave-like fashion. 


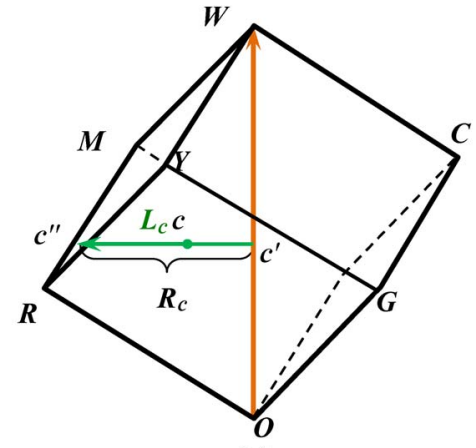

(a)

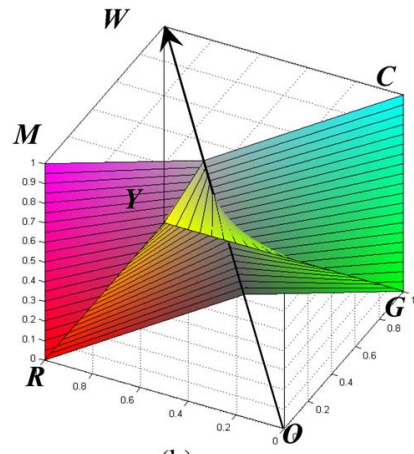

(b)

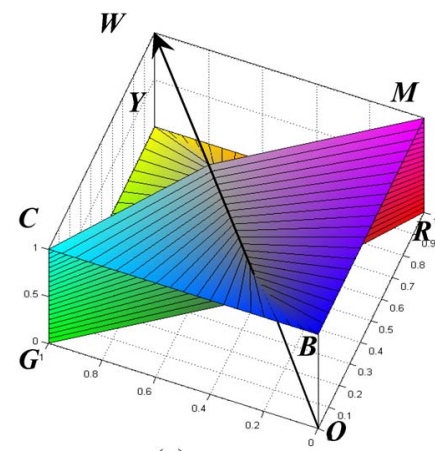

(c)
Fig. 3. Computation of surface boundary $B S_{\mathrm{RGB}}$. (a) Definition of maximum attainable saturation range. (b) Boundary surface $B S_{\mathrm{RGB}}$ between upper and lower halves seen from a viewpoint outside the RGB space. (c) Boundary surface $B S_{\mathrm{RGB}}$ seen from another viewpoint opposite to that of (b).

From Fig. 3(a), it is observed that one half of the RGB cube, $H_{\mathrm{CMY}}$, includes the upper three square patches $W M R Y$, $W Y G C$, and $W C B M$, and the other half, $H_{\mathrm{RGB}}$, includes the lower three ones $O R Y G, O G C B$, and $O B M R$. Accordingly, the idea behind the proposed scheme to find the boundary surface $B S_{\mathrm{RGB}}$ between the two halves $H_{\mathrm{CMY}}$ and $H_{\mathrm{RGB}}$ in the $\mathrm{RGB}$ space as shown in Fig. 2(f) is to penetrate horizontally through the RGB cube onto the gray axis $\overline{O W}$, with each penetrating ray starting from a color point located on the up-and-down wave of the six patch edges $\overline{R Y}, \overline{Y G}, \overline{G C}, \overline{C B}, \overline{B M}$, and $\overline{M R}$ on the cube surface and resulting in a horizontal saturation line. All such saturation lines together form the desired surface $B S_{\mathrm{RGB}}$, which separates the RGB cube into two totally symmetric parts, as shown in Fig. 2(f) or in Fig. 3(b) and (c). After $B S_{\mathrm{RGB}}$ is transformed into the iNIHS space, it becomes the desired surface $B S_{\text {iNIHS }}$ which cuts the iNIHS space into two symmetric parts $H_{\text {upper }}$ and $H_{\text {lower }}$ as shown in Fig. 2(g). The details of this scheme are described as an algorithm in the following.

Derivation of the description of the boundary surface between the two halves of the iNIHS space.

1) Calculate the intensity values of the six corners of the RGB cube, $R, G, B, C, Y$, and $M$, which have the six fixed hue angles $0^{\circ}, 120^{\circ}, 240^{\circ}, 60^{\circ}, 180^{\circ}$ and $300^{\circ}$, respectively.

2) By the principle of similar triangles, derive the intensity values of the color points on the patch edges $\overline{R Y}, \overline{Y G}$, $\overline{G C}, \overline{C B}, \overline{B M}$, and $\overline{M R}$ as follows while referring to Fig. 4 for the notations.

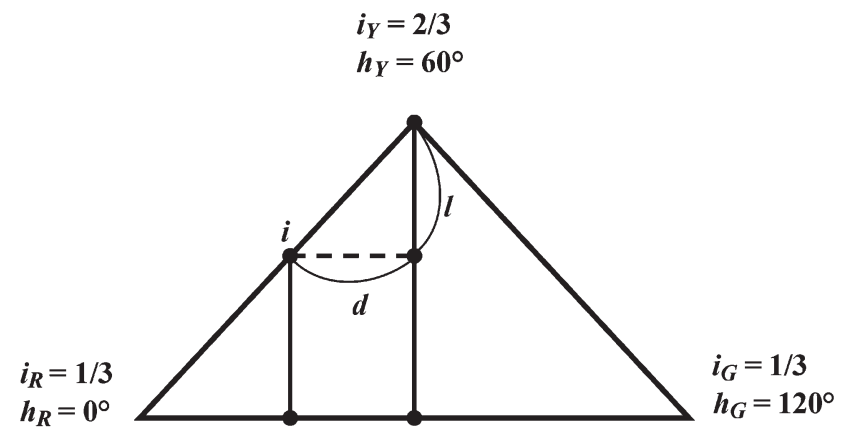

Fig. 4. Relationship between hue and intensity values for hue range $\left[0^{\circ}, 120^{\circ}\right]$.

2.1 Compute the intensity values of corners $R, G, B, C$, $Y$, and $M$ in Fig. 3(a) by the following steps.

A) Compute the intensity values $i_{R}, i_{G}$, and $i_{B}$ of corners $R, G$, and $B$, to be $i_{R}=i_{G}=$ $i_{B}=1 / 3$, according to (5), using the RGB values $c_{R}=(1,0,0), c_{G}=(0,1,0)$, and $c_{B}=$ $(0,0,1)$ of $R, G$, and $B$, respectively.

B) Compute similarly the intensity values $i_{C}, i_{M}$, and $i_{Y}$ of corners $C, M$, and $Y$, to be $i_{C}=$ $i_{M}=i_{Y}=2 / 3$ using the RGB values $c_{C}=$ $(0,1,1), c_{M}=(1,0,1)$, and $c_{Y}=(1,1,0)$ of $C, M$, and $Y$, respectively.

2.2 Divide the total hue range $\left[0,360^{\circ}\right]$ into six sections $H_{1}$ through $H_{6}$, each with an equal width of $60^{\circ}$, corresponding to $\overline{R Y}, \overline{Y G}, \overline{G C}, \overline{C B}, \overline{B M}$, and $\overline{M R}$, respectively.

2.3 For each color point $c=(r, g, b)$ on edge $\overline{R Y}$ other than $R$ and $Y$, compute its intensity $i$ in the following way while referring to Fig. 4 for an illustration.

A) Compute the absolute difference $d$ between the hue $h$ of $c$ and the hue $60^{\circ}$ of corner $Y$ as $d=$ $60^{\circ}-h$.

B) Compute the difference $l$ between the intensity of color point $c$ and that of corner $Y$ according to the principle of similar triangles, as $l=[(2 / 3-$ $\left.1 / 3) / 60^{\circ}\right] \times d=d / 180^{\circ}=\left(60^{\circ}-h\right) / 180^{\circ}$.

C) Compute the intensity $i$ of color point $c$ as $i=i_{Y}-l=2 / 3-\left(60^{\circ}-h\right) / 180^{\circ}$ where $h \in$ $\left[0^{\circ}, 60^{\circ}\right]$.

2.4 Compute similarly the intensity $i$ of a color point $c$ on the other five patch edges $\overline{Y G}, \overline{G C}, \overline{C B}, \overline{B M}$, and $\overline{M R}$, respectively, other than those of corners $R, G$, $B, C, Y$, and $M$ to be as follows:

$i=2 / 3-\left(h-60^{\circ}\right) / 180^{\circ}$ where $h \in\left[60^{\circ}, 120^{\circ}\right]$;

$i=2 / 3-\left(180^{\circ}-h\right) / 180^{\circ}$ where $h \in\left[120^{\circ}, 180^{\circ}\right]$;

$i=2 / 3-\left(h-180^{\circ}\right) / 180^{\circ}$ where $h \in\left[180^{\circ}, 240^{\circ}\right]$;

$i=2 / 3-\left(300^{\circ}-h\right) / 180^{\circ}$ where $h \in\left[240^{\circ}, 300^{\circ}\right]$;

$i=2 / 3-\left(h-300^{\circ}\right) / 180^{\circ}$ where $h \in\left[300^{\circ}, 360^{\circ}\right]$.

3) Combine the two results of Steps 2.3 and 2.4 for $\overline{R Y}$ and $\overline{Y G}$ as $i=2 / 3-\left|h-60^{\circ}\right| / 180^{\circ}$ where $h \in\left[0^{\circ}, 120^{\circ}\right]$. 
4) Perform similar operations for $\overline{G C}$ and $\overline{C B}$ to get $i=$ $2 / 3-\left|h-180^{\circ}\right| / 180^{\circ}$ where $h \in\left[120^{\circ}, 240^{\circ}\right]$; and for $\overline{B M}$ and $\overline{M R}$ to get $i=2 / 3-\left|h-300^{\circ}\right| / 180^{\circ}$ where $h \in\left[240^{\circ}, 360^{\circ}\right]$.

5) Combine the three results yielded in Steps 3 and 4 by normalizing the hue value $h$ to be $h_{\bmod } 120^{\circ}$ so that the intensity value $i$ may be rewritten in a unified form as

$$
i=\frac{2}{3}-\left|h_{\bmod 120^{\circ}}-60^{\circ}\right| / 180^{\circ} .
$$

6) Describe the boundary surface $B S_{\mathrm{iNIHS}}$ by

$$
i=2 / 3-\left|h_{\bmod 120^{\circ}}-60^{\circ}\right| / 180^{\circ}, 0 \leq s \leq 1
$$

where $(i, h, s)$ are the IHS values of a color point $c$ on $B S_{\text {iNIHS }}$.

On the other hand, the description of boundary surface $B S_{\mathrm{RGB}}$ in the RGB color space may be obtained by substituting (5) into (10) above.

\section{B. Proposed Improved RGB to Nonlinear IHS Conversion}

With (10) describing the boundary surface $B S_{\text {iNIHS }}$, we can now present the process for transforming a color point $c=$ $(r, g, b)$ in the RGB cube into the proposed iNIHS color space. At first, (5) is used to compute the intensity and hue values $i_{c}$ and $h_{c}$ of point $c$. Then, (10) is used to decide whether point $c$ is in the upper half, $H_{\text {upper }}$, or in the lower, $H_{\text {lower }}$, in the iNIHS space by the following way:

$$
\begin{array}{r}
\text { if } i_{c} \leq 2 / 3-\left|h_{c \bmod 120^{\circ}}-60^{\circ}\right| / 180^{\circ} \text {, then } c \text { is in } H_{\text {lower }} \text {; } \\
\text { else, in } H_{\text {upper }} \text {. }
\end{array}
$$

If $c$ is in $H_{\text {lower }}$, its saturation value $s_{\mathrm{c}}$ may be computed in the conventional way using (5) to be $s_{c}=1-3 \times$ $\min (r, g, b) /(r+g+b)$. And if $c$ is in $H_{\text {upper }}, s_{\mathrm{c}}$ should be, as mentioned previously, computed by a CMY to IHS transformation instead of by an RGB to IHS one. For this, the formula $s_{c}=$ $1-3 \times \min (r, g, b) /(r+g+b)$ may be used again except that the values of $r, g$, and $b$ should be replaced by $c, m$, and $y$, respectively, resulting in $s_{c}=1-3 \times \min (c, m, y) /(c+m+$ $y)$. But since we have $[c, m, y]^{T}=[1-r, 1-g, 1-b]^{T}$, we get, after some reductions, the following equivalent formula for $s_{c}$ :

$$
s_{c}=1-3 \times[1-\max (r, g, b)] /[3-(r+g+b)] .
$$

In summary, a complete set of equations for transforming a color point $c=(r, g, b)$ in the RGB space into the iNIHS space is listed as follows, which include the decision criterion of (11).

\section{RGB to iNIHS transformation.}

$$
\left\{\begin{array}{l}
a=\frac{(2 b-g-r) / 2}{\sqrt{(b-g)^{2}+(b-r)(g-r)}} ; i=\frac{r+g+b}{3} ; \\
\text { if } b \leq g, \text { then } h=\cos ^{-1}(a) ; \text { else } h=2 \pi-\cos ^{-1}(a) ; \\
\text { if } i \leq \frac{2}{3}-\frac{\mid h_{\bmod 120^{\circ}-60^{\circ} \mid}}{180^{\circ}} \\
\quad \text { then } s=1-\frac{3 \times \min (r, g, b)}{r+g+b} \text { (conventional); } \\
\quad \text { else } s=1-\frac{3(1-\max (r, g, b))}{3-(r+g+b)} \text { (improved). }
\end{array}\right.
$$

\section{Proposed Improved Nonlinear IHS to RGB Conversion}

After an image is generated by image fusion and processed in the iNIHS color space, conversion of the resulting image data back into the RGB space is necessary for image displays or other purposes. The formulas for the conventional nonlinear IHS to RGB transformation are shown in (6)-(8), in which the three sections of RG, GB, and BR are considered individually. These formulas can be used for each color point $c=(i, h, s)$ in the lower half $H_{\text {lower }}$ of the iNIHS space, which are repeated in the following except that the respective constraint of being in $H_{\text {lower }}$ for each section according to (10) is included.

Nonlinear IHS to RGB transformation for color points in the lower half $H_{\text {lower }}$ of the iNIHS space.

1) Section RG $\left(0^{\circ} \leq h<120^{\circ}\right.$ and $i \leq 2 / 3-\mid h-$ $\left.60^{\circ} \mid / 180^{\circ}\right)$ :

$$
\begin{aligned}
& b=i(1-s) ; r=i\left[1+s \cos h / \cos \left(60^{\circ}-h\right)\right] ; \\
& g=3 i-(b+r) .
\end{aligned}
$$

2) Section GB $\left(120^{\circ} \leq h<240^{\circ}\right.$ and $i \leq 2 / 3-\mid h-$ $\left.180^{\circ} \mid / 180^{\circ}\right)$ :

$$
\begin{aligned}
& h=h-120^{\circ} ; r=i(1-s) ; \\
& g=i\left[1+s \cos h / \cos \left(60^{\circ}-h\right)\right] ; b=3 i-(r+b) .
\end{aligned}
$$

3) Section BR $\left(240^{\circ} \leq h<360^{\circ}\right.$ and $i \leq 2 / 3-\mid h-$ $\left.300^{\circ} \mid / 180^{\circ}\right)$ :

$$
\begin{aligned}
& h=h-240^{\circ} ; g=i(1-s) ; \\
& b=i\left[1+s \cos h / \cos \left(60^{\circ}-h\right)\right] ; r=3 i-(g+b) .
\end{aligned}
$$

For color points in the upper half $H_{\text {upper }}$ of the iNIHS space, a back transformation into the CMY space should be applied, as mentioned previously. For this, the entire hue range of the iNIHS space is divided into three sections, CM, MY, and YC, which correspond to the sections RG, GB, and BR of the RGB space, respectively. To derive the formulas for these sections, it seems that we can just replace the values of $(r, g, b)$ in (6)-(8) with the values of $(c, m, y)$, respectively. But this is not enough —one additional operation is required, namely, to replace the intensity value $i$ in (6)-(8) with $1-i$ because the intensity value in the CMY space measured from its origin $W$ is just 1 minus that in the RGB space measured from the origin $O$ [see Fig. 3(a)]. Moreover, the sub-ranges of CM, MY, and $\mathrm{YC}$ are $\left[180^{\circ}, 300^{\circ}\right],\left[-60^{\circ}, 60^{\circ}\right]$, and $\left[60^{\circ}, 180^{\circ}\right]$, respectively, which are different from those of RG, GB, and $\mathrm{BR}$, namely, $\left[0^{\circ}\right.$, $\left.120^{\circ}\right],\left[120^{\circ}, 240^{\circ}\right]$ and $\left[240^{\circ}, 360^{\circ}\right]$, respectively.

Specifically, the sub-range of section CM, e.g., is $\left[180^{\circ}\right.$, $\left.300^{\circ}\right]$ which strides the two hue sections of $\left[180^{\circ}, 240^{\circ}\right]$ and $\left[240^{\circ}, 300^{\circ}\right]$. According to Steps 2.3 and 2.4 of the abovedescribed method for finding the boundary surface $B S_{\mathrm{iNIHS}}$, within the sub-range $\left[180^{\circ}, 240^{\circ}\right]$ the constraint for a color point to be in the upper half $H_{\text {upper }}$ of the iNIHS space is $i>2 / 3-\left(h-180^{\circ}\right) / 180^{\circ}$ and within $\left[240^{\circ}, 300^{\circ}\right]$ the constraint is $i>2 / 3-\left(300^{\circ}-h\right) / 180^{\circ}$. The two constraints 
can be combined to be a single one, namely, $i>1 / 3+\mid h-$ $240^{\circ} \mid / 180^{\circ}$. Similarly, the hue range of section MY is $\left[-60^{\circ}\right.$, $\left.60^{\circ}\right]$, which can be split into the two sub-ranges $\left[-60^{\circ}, 0^{\circ}\right]$ and $\left[0^{\circ}, 60^{\circ}\right]$ with the former being equivalent to $\left[300^{\circ}\right.$, $\left.360^{\circ}\right]$. Within $\left[300^{\circ}, 360^{\circ}\right]$, the constraint for a color point to be in the upper half $H_{\text {upper }}$ of the iNIHS space is $i>$ $2 / 3-\left(h-300^{\circ}\right) / 180^{\circ}$; and within $\left[0^{\circ}, 60^{\circ}\right]$, the constraint is $i>2 / 3-\left(60^{\circ}-h\right) / 180^{\circ}$. The two constraints are equivalent to $i>1 / 3+\left(360^{\circ}-h\right) / 180^{\circ}$ and $i>1 / 3+h / 180^{\circ}$, respectively. Finally, for the hue range $\left[60^{\circ}, 180^{\circ}\right]$ of $\mathrm{YC}$ consisting of the two sub-ranges $\left[60^{\circ}, 120^{\circ}\right]$ and $\left[120^{\circ}, 180^{\circ}\right]$, the corresponding constraints are $i>2 / 3-\left(h-60^{\circ}\right) / 180^{\circ}$ and $i>2 / 3-\left(180^{\circ}-h\right) / 180^{\circ}$, respectively. The two constraints may be combined to be a single one as well, namely, $i>$ $1 / 3+\left|h-120^{\circ}\right| / 180^{\circ}$. In summary, for a color point $c$ with color $(i, h, s)$ in the upper half $H_{\text {upper }}$ of the iNIHS space, the backward transformation of $c$ into the CMY space may be described as follows.

Nonlinear IHS to CMY transformation for color points in the upper half $\boldsymbol{H}_{\text {upper }}$ of the iNIHS space.

1) Section YC $\left(60^{\circ}<h \leq 180^{\circ}\right.$ and $i>1 / 3+\mid h-$ $\left.120^{\circ} \mid / 180^{\circ}\right)$ :

$$
\begin{aligned}
& h=h-240^{\circ} ; m=(1-i)(1-s) ; \\
& y=(1-i)\left[1+s \cos h / \cos \left(60^{\circ}-h\right)\right] ; \\
& c=3(1-i)-(m+y) .
\end{aligned}
$$

2) Section $\mathrm{CM}\left(180^{\circ}<h \leq 300^{\circ}\right.$ and $i>1 / 3+\mid h-$ $\left.240^{\circ} \mid / 180^{\circ}\right)$ :

$y=(1-i)(1-s) ; c=(1-i)\left[1+s \cos h / \cos \left(60^{\circ}-h\right)\right]$;

$m=3(1-i)-(c+y)$.

3) Section MY $\left(\left(-60^{\circ}<h \leq 0^{\circ}\right.\right.$ and $i>1 / 3+\left(360^{\circ}-\right.$ $\left.h) / 180^{\circ}\right)$ or $\left(0^{\circ}<h \leq 60^{\circ}\right.$ and $\left.\left.i>1 / 3+h / 180^{\circ}\right)\right)$ :

$$
\begin{aligned}
h & =h-120^{\circ} ; c=(1-i)(1-s) ; \\
m & =(1-i)\left[1+s \cos h / \cos \left(60^{\circ}-h\right)\right] ; \\
y & =3(1-i)-(c+m) .
\end{aligned}
$$

The final forms of the formulas for sections $\mathrm{YC}, \mathrm{CM}$, and MY in terms of the RGB color $(r, g, b)$ instead of the CMY color $(c, m, y)$ are summarized as follows, which are obtained by replacing $[c, m, y]^{T}$ with $[1-r, 1-g, 1-b]^{T}$, respectively. The details of the involved simplifications are omitted.

Nonlinear IHS to RGB transformation for color points in the upper half $H_{\text {upper }}$ of the iNIHS space.

1) Section YC $\left(60^{\circ}<h \leq 180^{\circ}\right.$ and $i>1 / 3+\mid h-$ $\left.120^{\circ} \mid / 180^{\circ}\right)$ :

$$
\begin{aligned}
& h=h-240^{\circ} ; g=i(1-s)+s ; \\
& b=1-(1-i)\left[1+s \cos h / \cos \left(60^{\circ}-h\right)\right] ; \\
& r=3 i-(g+b) .
\end{aligned}
$$

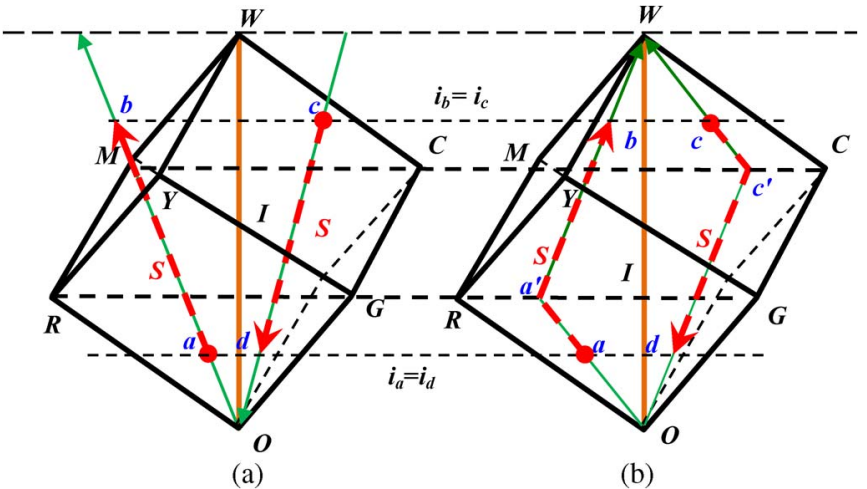

Fig. 5. Saturation variations in accordance with intensity changes. (a) Saturation change in nonlinear IHS space. (b) Saturation change in iNIHS space.

2) Section $\mathrm{CM}\left(180^{\circ}<h \leq 300^{\circ}\right.$ and $i>1 / 3+\mid h-$ $\left.240^{\circ} \mid / 180^{\circ}\right)$ :

$b=i(1-s)+s ; r=1-(1-i)\left[1+s \cos h / \cos \left(60^{\circ}-h\right)\right] ;$ $g=3 i-(b+r)$.

3) Section MY $\left(\left(-60^{\circ}<h \leq 0^{\circ}\right.\right.$ and $i>1 / 3+\left(360^{\circ}-\right.$ $\left.h) / 180^{\circ}\right)$ or $\left(0^{\circ}<h \leq 60^{\circ}\right.$ and $\left.\left.i>1 / 3+h / 180^{\circ}\right)\right)$ :

$$
\begin{aligned}
& h=h-120^{\circ} ; r=i(1-s)+s ; \\
& g=1-(1-i)\left[1+s \cos h / \cos \left(60^{\circ}-h\right)\right] ; \\
& b=3 i-(r+g) .
\end{aligned}
$$

\section{Saturation Variations in RGB Space after Intensity Substitution in the IHS Space}

As mentioned in Section I, an intensity substitution in the conventional nonlinear IHS space can be represented simply by a direct scaling operation in the RGB space [22], [23]. As a result, the saturation value is expanded outward as the substituting intensity is increased, as illustrated by Fig. 5(a), incurring a gamut problem when the substituting intensity value is too large. For example, as shown by the RGB cube in Fig. 5(a), if the intensity value of a color point $a$ [say, with $r_{a}=0.4, g_{a}=0.1$, and $b_{a}=0.1$; and so with $i_{a}=0.2, h_{a}=$ $0^{\circ}$, and $s_{a}=0.5$ according to (5)] is increased from $i_{a}$ to $i_{b}$ in the nonlinear IHS space with the hue and saturation kept unchanged, point $a$ will be "moved" to point $b$. Suppose that $i_{b}$ is equal to 0.8 . Then, according to (6) (for section RG), the RGB values at $b$ may be computed to be $r_{b}=1.6, g_{b}=0.4$, and $b_{b}=0.4$, which are out of the gamut because $r_{b}=1.6>1$, so that the corresponding intensity should be clipped to fit into the RGB cube. In image fusion, such a clipping will reduce the contrast of the final fusion result, as mentioned previously and as will be demonstrated by experimental results later.

On the other hand, if the intensity value of a color point $c$ [say, with $r_{c}=0.4, g_{c}=1$, and $b_{c}=1$; and so with $i_{c}=$ $0.8, h_{c}=180^{\circ}$, and $s_{c}=0.5$ according to (5)] in Fig. 5(a) is decreased from $i_{c}=i_{b}$ to $i_{d}=i_{a}$ in the nonlinear IHS space with the hue and saturation kept unchanged, point $c$ will be "moved" to point $d$. Because $i_{d}$ is equal to 0.2 , according to 
(7) (for section $\mathrm{GB}$ ), the RGB values at $d$ may be computed to be $r_{d}=0.1, g_{d}=0.25$, and $b_{d}=0.25$, which causes no gamut problem. However, the saturation component is scaled down because $i_{d}$ is smaller than $i_{c}$. This means that in image fusion, if the intensity of the PAN image is much lower than the MS image, the color of the fusion result will be de-saturated, resulting in color distortion.

In contrast, in the proposed iNIHS color space, the saturation component varies adaptively within the maximum attainable saturation range of the RGB cube without causing the gamut problem. This improvement may be observed from two parts. Below the boundary surface $B_{\mathrm{RGB}}$ as mentioned in Section III, the saturation component is scaled up as in the conventional nonlinear IHS space according to (12)-(14). On the other hand, above the boundary surface $B S_{\mathrm{RGB}}$, in section MY, e.g., if the intensity $i$ of a color point is substituted by $i^{\prime}$, the new CMY color values can be derived from (19) to be

$$
\left[\begin{array}{c}
c^{\prime} \\
m^{\prime} \\
y^{\prime}
\end{array}\right]=\left[\begin{array}{l}
\left(1-i^{\prime}\right)(1-s) \\
\left(1-i^{\prime}\right)\left[1+s \cos h / \cos \left(60^{\circ}-h\right)\right] \\
3\left(1-i^{\prime}\right)-\left(c^{\prime}+m^{\prime}\right)
\end{array}\right]=\gamma\left[\begin{array}{c}
c \\
m \\
y
\end{array}\right]
$$

where $\gamma=\left(1-i^{\prime}\right) /(1-i)$. Substituting $[c, m, y]^{T}$ by $[1-$ $r, 1-g, 1-b]^{T},\left[c^{\prime}, m^{\prime}, y^{\prime}\right]^{T}$ by $\left[1-r^{\prime}, 1-g^{\prime}, 1-b^{\prime}\right]^{T}$, and simplifying the result, we can get

$$
\left[r^{\prime}, g^{\prime}, b^{\prime}\right]^{T}=[1,1,1]^{T}-\gamma \times[1-r, 1-g, 1-b]^{T} .
$$

For sections $\mathrm{YC}$ and $\mathrm{CM}$, similar formulas may be derived using (17) and (18).

For example, as shown by the RGB cube seen in Fig. 5(b), if the intensity value of a color point $a$ [say, with $r_{a}=0.4$, $g_{a}=0.1$ and $b_{a}=0.1$; and so with $i_{a}=0.2, h_{a}=0^{\circ}$, and $s_{a}=0.5$ according to (13)] is increased from $i_{a}$ to $i_{b}$ in the iNIHS color space with the hue and saturation values kept unchanged, the color value will firstly be scaled up in the range below the boundary surface $B S_{\text {iNIHS }}$ so that the color point is "moved" to point $a^{\prime}$, and then be scaled down in the range above $B S_{\mathrm{iNIHS}}$ so that the color point is "moved" further to point $b$. Supposed that $i_{b}$ is equal to 0.8 . Then, according to (22) (for section MY), the RGB values at point $b$ may be computed to be $r_{b}=0.9, g_{b}=0.75$, and $b_{b}=0.75$. No gamut problem occurs here, in contrast with the result of using the conventional transformations mentioned above.

On the other hand, if the intensity of a color point $c$ [say, with $r_{c}=0.4, g_{c}=1$, and $b_{c}=1$; and so with $i_{c}=0.8, h_{c}=180^{\circ}$, and $s_{c}=1$ according to (13)] is decreased from $i_{c}=i_{b}$ to $i_{d}=i_{a}$ in the iNIHS color space with the hue and saturation kept unchanged, the color value will firstly be scaled up in the range above $B S_{\mathrm{iNIHS}}$ so that the color point is "moved" to point $c^{\prime}$, and then be scaled down in the range below $B S_{\mathrm{iNIHS}}$ so that the color point is "moved" to point $d$. Because $i_{d}$ is equal to 0.2 , according to (15) (for section GB), the RGB values may be computed to be $r_{d}=0, g_{d}=0.3$, and $b_{d}=0.3$. It is so concluded that for image fusion in the proposed iNIHS color space, all desired intensity substitutions can be achieved with no gamut problem incurred if the intensity and saturation components are manipulated within the range of 0 to 1 .

\section{E. Proposed Method for Solving the Gamut Problem}

In summary, with the input of an MS image $M S$ and a PAN image Pan both with RGB color values, the proposed method for solving the gamut problem without appealing to clipping may be described as an algorithm as follows.

1) Forward transforming MS into the iNIHS color space. For each pixel $p$ in $M S$, decide which half, $H_{R G B}$ or $H_{\mathrm{CMY}}$, of the RGB color space the color point $c$ specified by the RGB values of $p$ falls into, and conduct the corresponding RGB to IHS transformation described by (13) in Section III-B, resulting in a new MS image denoted as $M S_{\text {IHS }}$.

2) Conducting image fusion operations. Perform necessary image processing operations in the improved nonlinear IHS color space (e.g., substituting the intensity values of pixels in $M S_{\mathrm{IHS}}$ with those of the corresponding pixels in image Pan), and denote the resulting image as $M S_{\mathrm{IHS}}^{\prime}$.

3) Backward transforming the processed image $M_{\mathrm{IHS}}^{\prime}$ into the RGB space. For each pixel $p^{\prime}$ in $M S_{\mathrm{IHS}}^{\prime}$, decide which half, $H_{\text {upper }}$ or $H_{\text {lower }}$, the color point $c^{\prime}$ specified by the IHS values of $p^{\prime}$ falls into, and conduct the corresponding IHS to RGB transformation described by (14)-(16) or by (20) through (26), respectively, resulting in a new MS image $M S^{\prime}$ with RGB color values as the output.

\section{EXPERIMENTAL RESUlts AND Discussions}

\section{A. Visual Analysis}

Two major problems, namely, contrast reduction caused by the gamut problem and saturation degradation caused by intensity decreasing, will be demonstrated by experimental results of image fusion at first in this section. Artificial PAN and MS images were generated from given color satellite images to test the effect of the proposed image fusion method. The generation process includes three major steps: 1) transform a given color satellite image $I$ into a grayscale one $G$, equalize the histogram of $G$, and take the result as a PAN image; 2) down sample $I$ to be with $1 / 16$ of its original resolution, resulting in another image $I^{\prime}$; and 3) darken $I^{\prime}$ so that the intensity values of the PAN image are mostly higher than those of the resulting image, which then is taken as an MS image. Here, Step 3 is conducted for the purpose of evaluating the gamut problem caused by substitutions of higher intensity values into the MS image. An example of the results of this process is shown in Fig. 6 where Fig. 6(a) is the generated PAN image and Fig. 6(b) the generated MS image.

After substitutive image fusion in the conventional linear IHS color space was conducted, the resulting image is shown in Fig. 6(c), which has better details than the original MS image except in the blue-colored regions, where most of the pixels are out of the gamut, as indicated by the white pixels shown in Fig. 6(d). Here, when generating Fig. 6(c), intensity clipping has been applied to the processed MS image in the IHS space before the image was transformed back into the RGB color space. In addition, the substitutive image fusion 


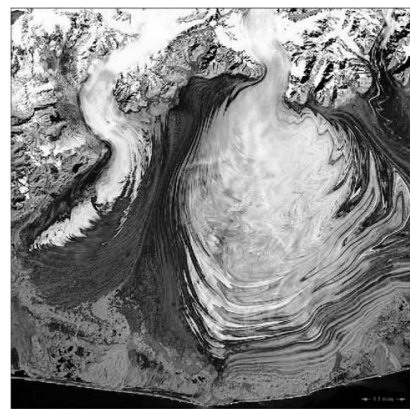

(a)

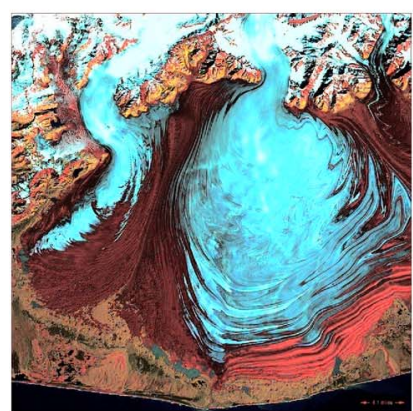

(c)

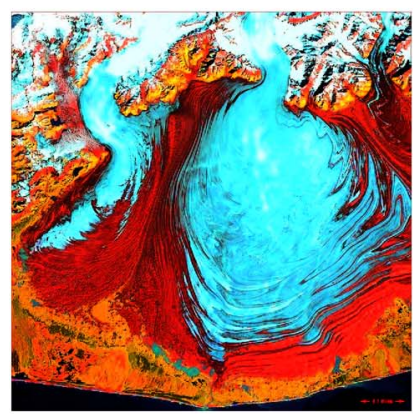

(e)

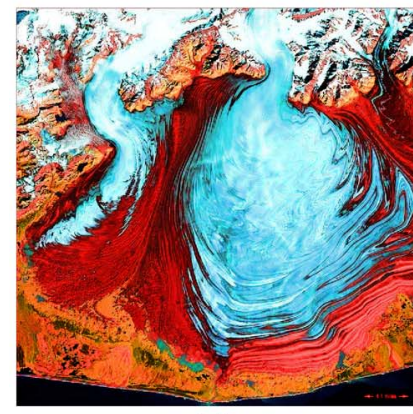

(g)

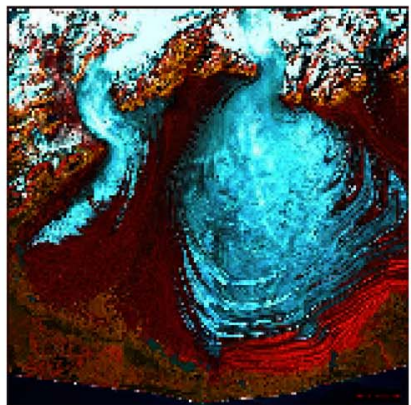

(b)

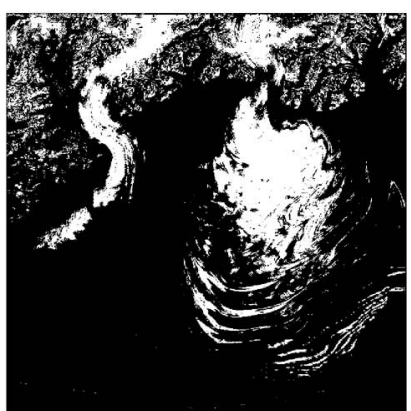

(d)

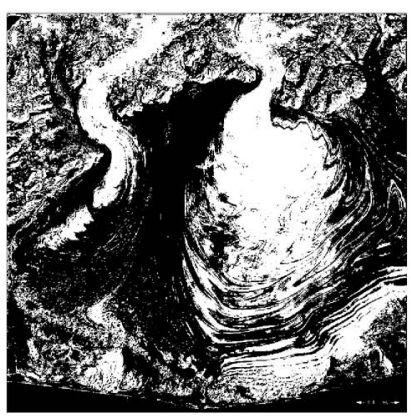

(f)

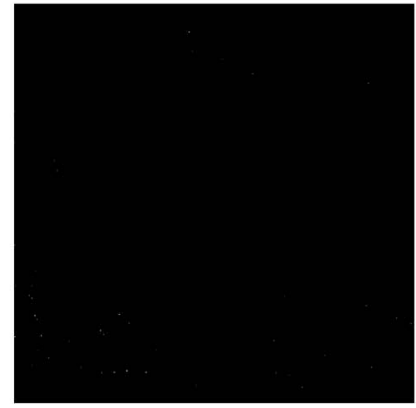

(h)
Fig. 6. Image fusion results of a PAN image with a dark MS image. (a) PAN image obtained from a given color satellite image by gray transformation, histogram equalization, and darkening. (b) MS image obtained from down sampling the given image. (c) Fusion result of (a) and (b) in linear IHS color space. (d) Out-of-gamut pixels in linear IHS color space displayed in white. (e) Fusion result of (a) and (b) in conventional nonlinear IHS color space. (f) Out-of-gamut pixels in conventional nonlinear IHS color space displayed in white. (g) Fusion result of (a) and (b) in iNIHS color space. (h) No out-ofgamut pixel yielded except a very few caused by truncations.

operation was also conducted in the conventional nonlinear IHS space, and the result is shown in Fig. 6(e) which may be seen to have better colors and details than Fig. 6(c) because of the enhancement of the saturation components. But, similarly, many pixels of the processed MS image in the nonlinear IHS space are out of the gamut, as indicated by the white pixels shown in Fig. 6(f). And again, clipping had been done to them before Fig. 6(e) was generated. Finally, the proposed method for substitutive image fusion in the iNIHS space was applied, resulting in Fig. 6(g), which can be seen to preserve even more details because the colors are not oversaturated. Specifically, after intensity substitutions in the iNIHS space were conducted, all the colors are preserved within the gamut except a very few ones caused by truncations during the color transformations, as indicated by the white dots shown in Fig. 6(h).

Also, conducted was an experiment similar to the previous one except that the generated MS image was brightened, instead of darkened, as shown in Fig. 7(b). After intensity substitutions in the linear and nonlinear IHS spaces were conducted, most image details are preserved as shown in Fig. 7(c) and (e), respectively, since the out-of-gamut pixels are sparser now than the results of the last experiment, as indicated by the white pixels shown in Fig. 7(d) and (f), respectively. However, the substituting intensity values were again clipped if they were out of the gamut. On the other hand, after intensity substitutions in the iNIHS color space were conducted, not only the contrast details but also the saturation values are preserved, as shown in Fig. 7(g). Again, Fig. 7(h) indicates that no transformed color is out of the gamut except a few ones caused by truncations.

\section{B. Quantitative Analysis}

We have evaluated the intensity differences between the image fusion results of Figs. 6 and 7 and the PAN image caused by the gamut problem by the measures of correlation coefficient (CC) and root-mean-square error (RMSE). The result is shown in Table I from which we can see that RGB or intensity clipping causes the $\mathrm{CC}$ of the intensity component to be smaller than 1 and the RMSE to be larger than 0, in contrast with the $\mathrm{CC}$ and RMSE values resulting from the image generated by image fusion in the iNIHS space, which are equal to 1 and 0 , respectively, because no clipping was applied in generating the fusion results whose intensity values are totally conformed to those of the PAN image.

\section{Improvement on Color Distortions Caused by Intensity Differences Between PAN and MS Images}

It is noted that the proposed iNIHS color space can only resolve color distortions caused by the gamut problem; it does not solve color distortions caused by large intensity differences between the original PAN and the MS images. This phenomenon can be seen from Fig. 6(b) and (g), where the former, which is the MS image, is darker while the latter, which is the result yielded by the proposed method, is brighter. Fig. 7(b) and (g) show the same phenomenon. Such a color distortion phenomenon can be explained in the following way. Look at Fig. 5(b) and notice that if the intensity component of a pixel $p$ is substituted by a value in the range of $i_{a}$ to $i_{b}$ in the proposed iNIHS space, then although the gamut problem can be avoided, yet the saturation component in the meantime 


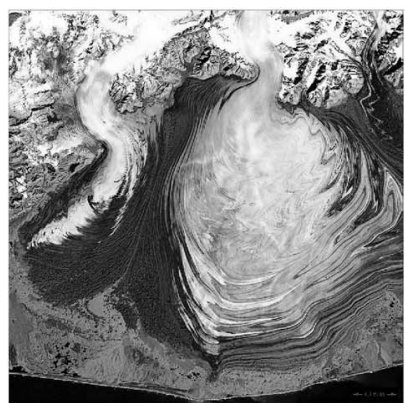

(a)

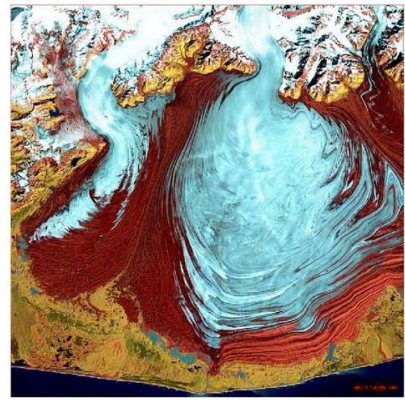

(c)

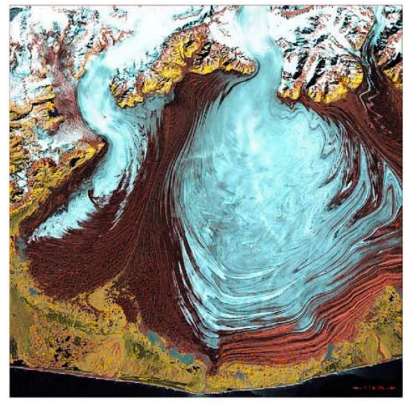

(e)

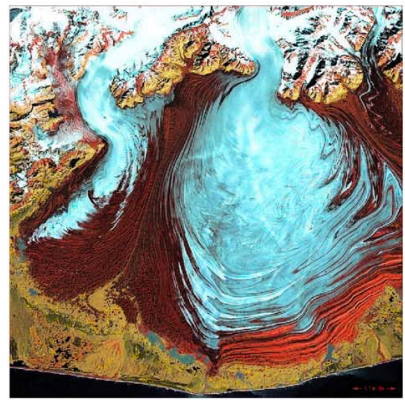

(g)

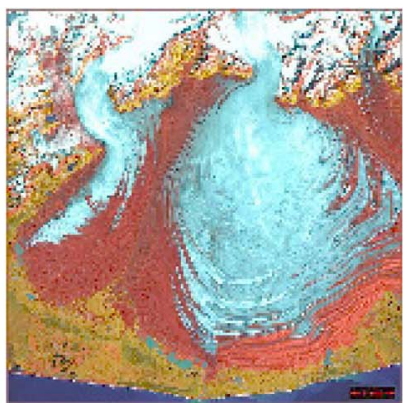

(b)

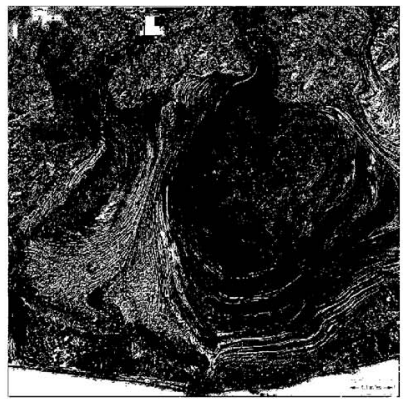

(d)

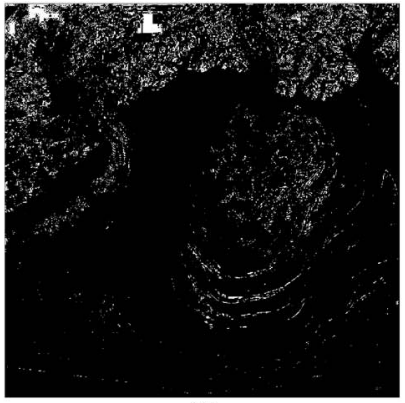

(f)

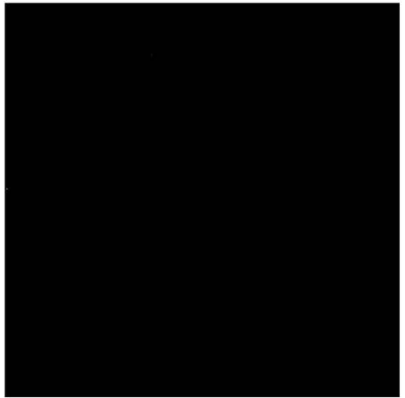

(h)
Fig. 7 Image fusion results of a PAN image with a bright MS image (a) PAN image obtained from a given color satellite image by gray transformation, histogram equalization, and brightening. (b) MS image obtained from down sampling the given image. (c) Fusion result of (a) and (b) in linear IHS color space. (d) Out-of-gamut pixels in linear IHS color space displayed in white. (e) Fusion result of (a) and (b) in conventional nonlinear IHS color space (f) Out-of-gamut pixels in conventional nonlinear IHS color space displayed in white. (g) Fusion result of (a) and (b) in iNIHS color space. (h) No out-of-gamut pixel yielded except a very few caused by truncations.

is reduced, resulting in color distortion. Notice also that in the proposed iNIHS color space, if the substituted intensity $i_{b}$ of pixel $p$ is very high, the original color $c_{p}$ of $p$ will be transformed undesirably to be totally white; and if $i_{b}$ is very low, $c_{p}$ will be transformed undesirably as well to be totally black, as can be seen from Fig. 5(b).
TABLE I

Difference Between Fusion Result and Pan IMAGE

\begin{tabular}{|c|c|c|c|}
\hline & Fusion Methods & $\begin{array}{l}\text { Correlation } \\
\text { Coefficient }\end{array}$ & $\begin{array}{l}\text { Root-mean- } \\
\text { square Error }\end{array}$ \\
\hline Dark & Fusion in linear IHS space (RGB Clipping) & 0.9996 & 0.0192 \\
\hline MS & Fusion in linear IHS space (intensity clipping) & 0.9983 & 0.0390 \\
\hline image & Fusion in IHS nonlinear space (RGB Clipping) & 0.9971 & 0.0537 \\
\hline & Fusion in nonlinear IHS space (intensity clipping) & 0.9948 & 0.0733 \\
\hline Fig. 6) & Fusion in iNIHS space (no clipping) & 1.0000 & 0 \\
\hline & Fusion in linear IHS space (RGB Clipping) & 0.9998 & 0.0114 \\
\hline MS & Fusion in linear IHS space (intensity clipping) & 0.9989 & 0.0278 \\
\hline image & Fusion in IHS nonlinear space (RGB Clipping) & 0.9999 & 0.0055 \\
\hline & Fusion in nonlinear IHS space (intensity clipping) & 0.9998 & 0.0108 \\
\hline Fig & Fusion in iNIHS space (no clipping) & 1.0000 & 0 \\
\hline
\end{tabular}

To resolve this problem, one way is to keep the intensity component of the MS image roughly unaltered and inject only the spatial detail information in the PAN image into the MS image during the image fusion process. For this purpose, the à trous wavelet algorithm [17] was adopted to extract the highfrequency spatial detail information in the PAN image, which is then added into the intensity component of the MS image. That is, the substitutive image fusion scheme of replacing the intensity components of the MS image with those of the PAN image as depicted in Fig. 1 is now changed to be an additive image fusion scheme of adding the high-frequency details of the intensity components of the PAN image to those of the MS image. In more detail, instead of using the substitutive image fusion algorithm described in Section III-E, we now adopt the following additive version for image fusion.

1) Extracting details from the PAN image. Smooth PAN image Pan by using the à trous wavelet filter [17], resulting in a smoothed PAN image $P a n^{\prime}$; and obtain an image $P_{d}$ of the detail information in $P$ an by subtracting Pan' from Pan.

2) Forward transforming MS into the iNIHS color space. For each pixel $p$ in $M S$, decide which half, $H_{R G B}$ or $H_{\mathrm{CMY}}$, of the RGB color space the color point $c$ specified by the RGB values of $p$ falls into, and conduct the corresponding RGB to IHS transformation described by (13) in Section III-B, resulting in a new MS image denoted as $M S_{\mathrm{IHS}}$

3) Conducting image fusion operations. Add the values of the pixels of $P_{d}$ to the intensity values of the corresponding pixels in $M S_{\mathrm{IHS}}$ and denote the resulting image as $M S_{\mathrm{IHS}}^{\prime}$.

4) Backward transforming the processed image $M S_{\mathrm{IHS}}^{\prime}$ into the RGB space. For each pixel $p^{\prime}$ in $M S_{\mathrm{IHS}}^{\prime}$, decide which half, $H_{\text {upper }}$ or $H_{\text {lower }}$, the color point $c^{\prime}$ specified by the IHS values of $p^{\prime}$ falls into, and conduct the corresponding IHS to RGB transformation described by (14)-(16) or by (20) through (26), respectively, resulting in a new MS image $M S^{\prime}$ with RGB color values as the output.

We have applied the above additive image fusion algorithm to Fig. 6(a) and (b) as well as Fig. 7(a) and (b) to yield two new fusion results as shown in Fig. 8(a) and (d), respectively. For comparisons, we also include the previous results of substitutive image fusion, Figs. 6(g) and 7(g), into Fig. 8 as Fig. 8(b) 


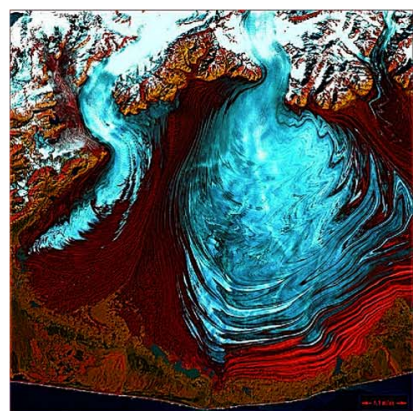

(a)

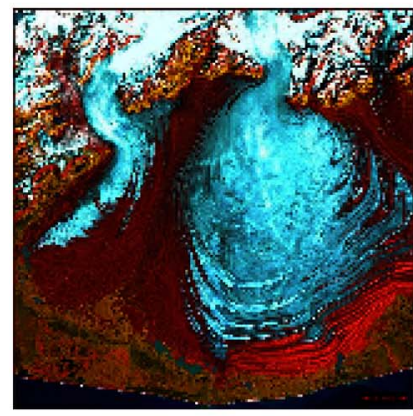

(c)

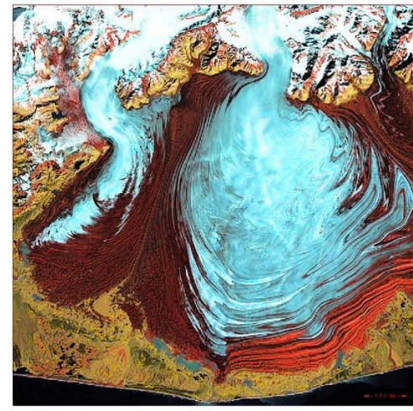

(e)

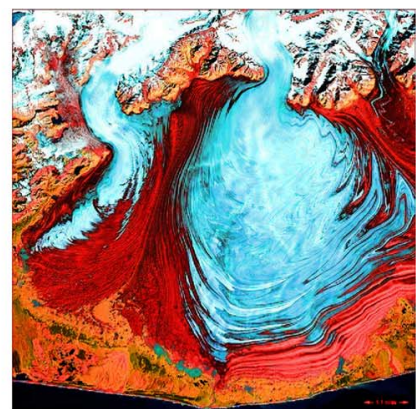

(b)

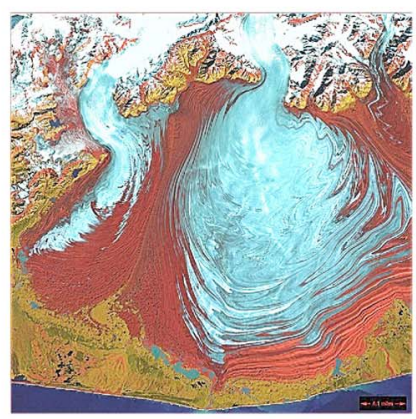

(d)

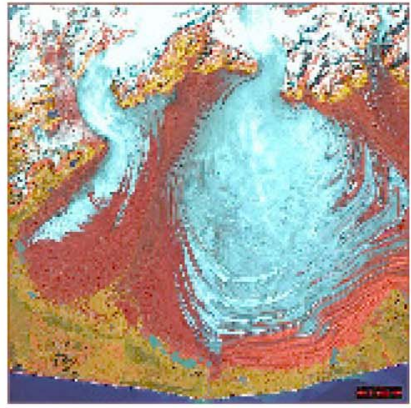

(f)
Fig. 8. Comparison of results of substitutive and additive Image fusions. (a) Additive fusion result from use of images in Fig. 6(a) and (b). (b) Former substitutive fusion result from use of Fig. 6(a) and (b). (c) Original MS image shown in Fig. 6(b). (d) Additive fusion result from use of images in Fig. 7(a) and (b). (e) Former substitutive fusion result from use of Fig. 7(a) and (b). (f) Original MS image shown in Fig. 7(b).

and (e), respectively. As can be seen now, the colors of the new results are closer to those of the original MS images Figs. 6(b) and 7(b), respectively, which are also included in Fig. 8 as Fig. 8(c) and (f). This means that the additive image fusion algorithm indeed keeps the original color appearance of the MS image more effectively than the substitutive algorithm.

To make the experimental results more convincing using real satellite images, two pairs of PAN and MS images acquired by satellites QuickBird and Ikonos as shown in Fig. 9(a) and (b), and Fig. 10(a) and (b), respectively, were used to conduct experiments similar to those described above, including substitutive and additive image fusions. The results are shown in Fig. 9(c)-(j), and in Fig. 10(c)-(j), respectively, from which conclusions similar to those drawn previously can be reached. Specifically, as shown by Figs. 9(i) and 10(i), the color appearances of the MS images are kept better in the results of additive image fusion than in those of substitutive image fusion.

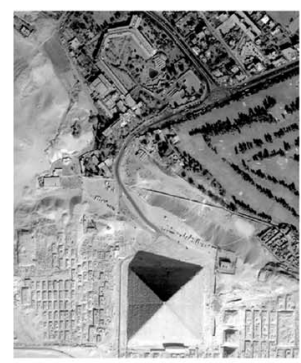

(a)

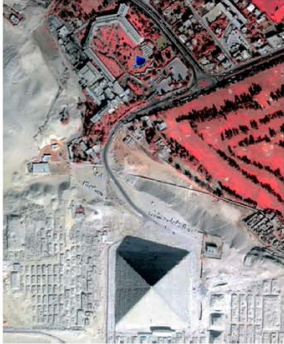

(c)

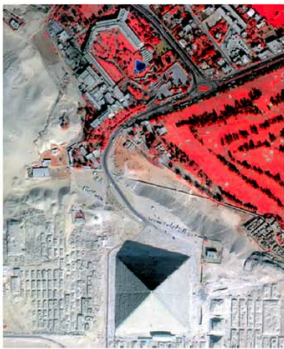

(e)

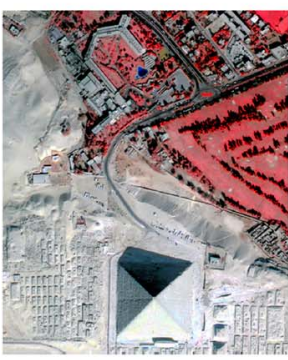

(g)

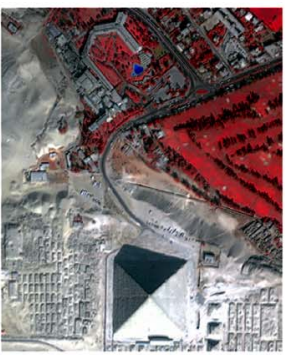

(i)

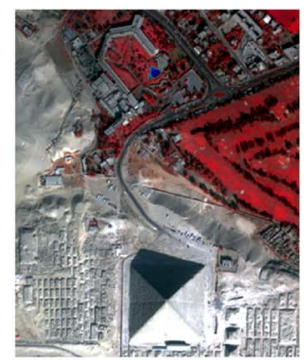

(b)

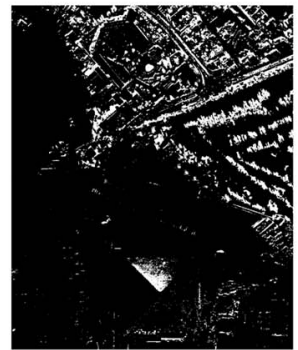

(d)

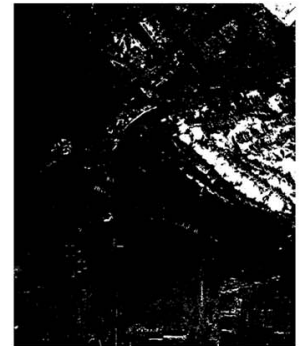

(f)

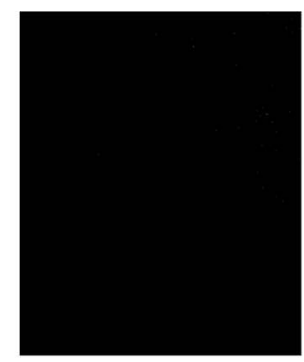

(h)

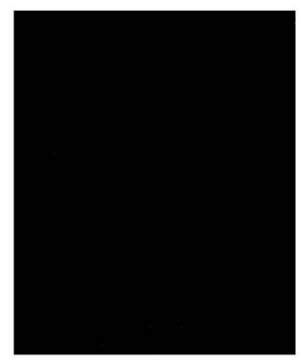

(j)
Fig. 9. Image fusion results of a PAN and an MS image acquired by satellite QuickBird. (a) PAN image obtained from a given color satellite image by gray transformation, histogram equalization, and darkening. (b) MS image obtained from down sampling the given image. (c) Fusion result of (a) and (b) in linear IHS color space using substitutive image fusion. (d) Out-of-gamut pixels in linear IHS color space displayed in white. (e) Fusion result of (a) and (b) in conventional nonlinear IHS color space using substitutive image fusion. (f) Outof-gamut pixels in conventional nonlinear IHS color space displayed in white. (g) Fusion result of (a) and (b) in proposed iNIHS color space using substitutive image fusion. (h) No out-of-gamut pixel yielded except a very fewcaused by truncations. (i) Fusion result of (a) and (b) in proposed iNIHS color space using additive image fusion. (j) No out-of-gamut pixel yielded except a very fewcaused by truncations. 


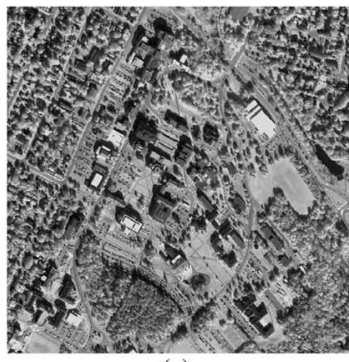

(a)

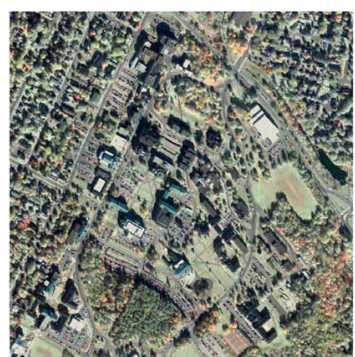

(c)

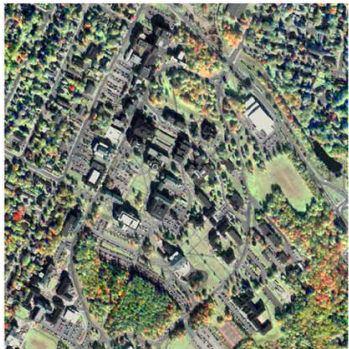

(e)

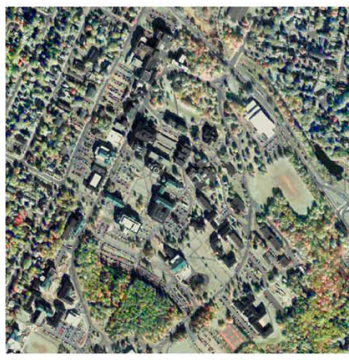

(g)

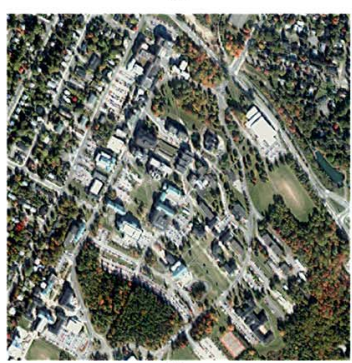

(i)

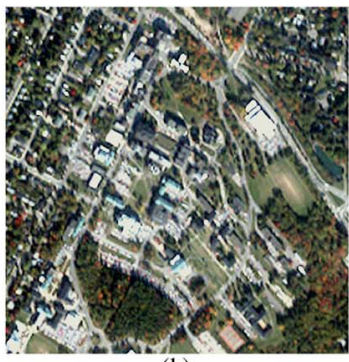

(b)

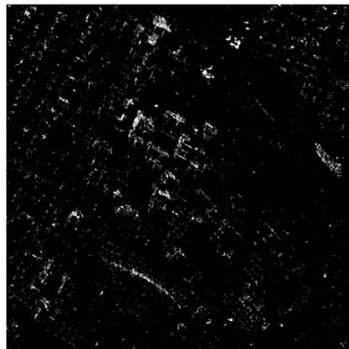

(d)

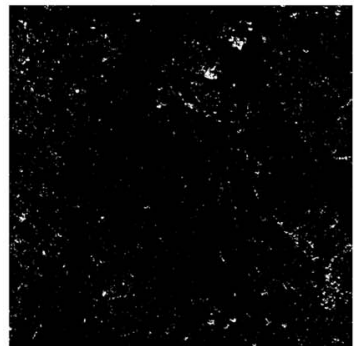

(f)

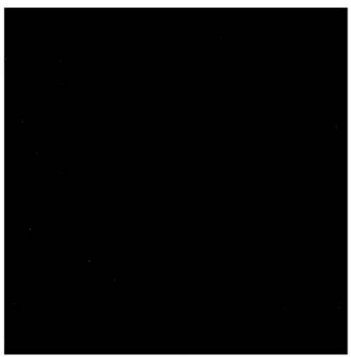

(h)

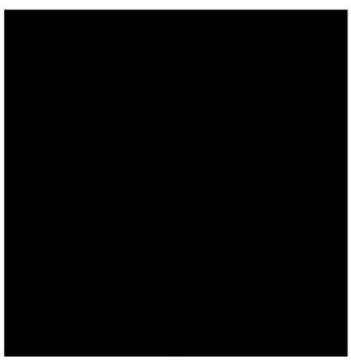

(j)
Fig. 10. Image fusion results of a PAN and an MS image acquired by satellite Ikonos. (a) PAN image obtained from a given color satellite image by gray transformation, histogram equalization, and darkening. (b) MS image obtained from down sampling the given image. (c) Fusion result of (a) and (b) in linear IHS color space using substitutive image fusion. (d) Out-of-gamut pixels in linear IHS color space displayed in white. (e) Fusion result of (a) and (b) in conventional nonlinear IHS color space using substitutive image fusion. (f) Outof-gamut pixels in conventional nonlinear IHS color space displayed in white. (g) Fusion result of (a) and (b) in proposed iNIHS color space using substitutive image fusion. (h) No out-of-gamut pixel yielded except a very fewcaused by truncations. (i) Fusion result of (a) and (b) in proposed iNIHS color space using additive image fusion. (j) No out-of-gamut pixel yielded except a very few caused by truncations.

\section{CONCLUSiOn AND Discussions}

Both the conventional linear and nonlinear transformations between the RGB and IHS color spaces incur gamut problems when used for image fusion. Clipping is usually used to solve the problem, leading to undesired color distortions and contrast reductions in the fusion result. An iNIHS color space has been proposed to solve the gamut problem without appealing to clipping. Equations describing the boundary surface between the two halves of the iNIHS space, and those describing the forward and backward transformations between the RGB and iNIHS spaces have also been derived for use in image fusion. Accordingly, not only the gamut problem is solved, but also the saturation component is adjusted adaptively within the maximum attainable saturation range. Consequently, all desired intensity substitutions during the image fusion process can be achieved. Moreover, the saturation components will not be reduced if the substituting intensity is decreased. Finally, if the intensity difference between the PAN image and the MS image is large, then even if the gamut problem is solved by the use of the iNIHS space, color distortions will still occur. In such cases, additive image fusion instead of substitutive fusion should be applied, as conducted in this study. Future studies may be directed to enhancing the proposed method to preserve multispectral image characteristics more effectively, and applying the method to works other than image fusion.

\section{REFERENCES}

[1] C. K. Munechika, J. S. Warnick, C. Salvaggio, and J. R. Schott, "Resolution enhancement of multispectral image data to improve classification accuracy," Photogramm. Eng. Remote Sens., vol. 59, no. 1, pp. 67-72, Jan. 1993

[2] Y. Zhang, "Understanding image fusion," Photogramm. Eng. Remote Sens., vol. 70, no. 6, pp. 657-661, 2004.

[3] M. Gonzalez-Audicana, J. L. Saleta, R. G. Catalan, and R. Garcia, "Fusion of multispectral and panchromatic images using improved IHS and PCA mergers based on wavelet decomposition," IEEE Trans. Geosci. Remote Sens., vol. 42, no. 6, pp. 1291-1299, Jun. 2004.

[4] Z. Wang, D. Ziou, C. Armenakis, D. Li, and Q. Li, "A comparative analysis of image fusion methods," IEEE Trans. Geosci. Remote Sens., vol. 43, no. 6, pp. 1391-1402, Jun. 2005.

[5] C. Thomas, T. Ranchin, L. Wald, and J. Chanussot, "Synthesis of multispectral images to high spatial resolution: Acritical review of fusion methods based on remote sensing physics," IEEE Trans. Geosci. Remote Sens., vol. 46, no. 5, pp. 1301-1312, May 2008.

[6] E. M. Schetselaar, "Fusion by the IHS transform: Should we use cylindrical or spherical coordinates?" Int. J. Remote Sens., vol. 19, no. 4, pp. 759765, Mar. 1998

[7] T. M. Tu, S. C. Su, H. C. Shyu, and P. S. Huang, "A new look at IHS-like image fusion methods," Inf. Fusion, vol. 2, no. 3, pp. 177-186, Sep. 2001.

[8] G. Xiao, Z. Jing, J. Li, and H. Leung, "Analysis of color distortion and improvement for IHS image fusion," in Proc. IEEE Intell. Transp. Syst., 2003, vol. 1, pp. 80-85.

[9] J. A. Malpica, "Hue adjustment to IHS Pan-sharpened IKONOS imagery for vegetation enhancement," IEEE Geosci. Remote Sens. Lett., vol. 4, no. 1, pp. 27-31, Jan. 2007.

[10] Y. Kim, Y. Eo, Y. Kim, and Y. Kim, "Generalized IHS-based satellite imagery fusion using spectral response functions," ETRI J., vol. 33, no. 4, pp. 497-505, Aug. 2011.

[11] F. Chen, F. Qin, G. Peng, and S. Chen, "Fusion of remote sensing images using improved ICA mergers based on wavelet decomposition," Proc. Eng., vol. 29, pp. 2938-2943, 2012.

[12] P. S. J. Chavez, S. C. Sides, and J. A. Anderson, "Comparison of three different methods to merge multiresolution and multispectral data: LANDSAT TM and SPOT panchromatic," Photogramm. Eng. Remote Sens., vol. 57, no. 3, pp. 295-303, Mar. 1991. 
[13] S. Zheng, W. Z. Shi, J. Liu, and J. Tian, "Remote sensing image fusion using multiscale mapped LS-SVM," IEEE Trans. Geosci. Remote Sens., vol. 46, no. 5, pp. 1313-1322, May 2008.

[14] J. Nunez, X. Otazu, O. Fors, A. Prades, V. Pala, and R. Arbiol, "Multiresolution-based image fusion with additive wavelet decomposition," IEEE Trans. Geosci. Remote Sens., vol. 37, no. 3, pp. 1204-1211, May 1999.

[15] B. Aiazzi, L. Alparone, S. Baronti, and A. Garzelli, "Context-driven fusion of high spatial and spectral resolution images based on oversampled multiresolution analysis," IEEE Trans. Geosci. Remote Sens., vol. 40, no. 10, pp. 2300-2312, Oct. 2002.

[16] G. Pajares and J. Manuel de la Cruz, "A wavelet-based image fusion tutorial," Pattern Recognit., vol. 37, no. 9, pp. 1855-1872, Sep. 2004.

[17] M. González-Audícana, X. Otazu, O. Fors, and A. Seco, "Comparison between Mallat's and the 'a trous' discrete wavelet transform based algorithms for the fusion of multispectral and panchromatic images," Int. J. Remote Sens., vol. 26, no. 3, pp. 595-614, Feb. 2005.

[18] S. H. Chen, H. Su, R. Zhang, and J. Tian, "Fusing remote sensing images using a'trous wavelet transform and empirical mode decomposition," Pattern Recognit. Lett., vol. 29, no. 3, pp. 330-342, Feb. 2008.

[19] C. Wang, P. Bong, J. Li, and Y. Zhang, "A United $l \alpha \beta$-contourlet method for remote sensing image fusion," in Proc. IEEE Int. Conf. Spatial Data Mining Geograph. Knowl. Serv., 2011, pp. 340-342.

[20] L. Alparone, L. Wald, J. Chanussot, C. Thomas, P. Gamba, and L. M. Bruce, "Comparison of pansharpening algorithms: Outcome of the 2006 GRS-S data-fusion contest," IEEE Trans. Geosci. Remote Sens., vol. 45, no. 10, pp. 3012-3021, Oct. 2007.

[21] C. C. Yang and S. H. Kwok, "Efficient gamut clipping for color image processing using LHS and YIQ," Opt. Eng., vol. 42, no. 3, pp. 701-711, Mar. 2003.

[22] C. C. Yang and J. J. Rodriguez, "Efficient luminance and saturation processing techniques for bypassing color coordinate transformations," in Proc. IEEE Int. Conf. Syst., Man, Cybern., 1995, vol. 1, pp. 667-672.

[23] C. C. Yang and J. J. Rodriguez, "Saturation clipping in the LHS and YIQ color spaces," Proc. SPIE, vol. 2658, no. 1, pp. 297-307, 1996.

[24] M. Choi, "A new intensity-hue-saturation fusion approach to image fusion with a tradeoff parameter," IEEE Trans. Geosci. Remote Sens., vol. 44, no. 6, pp. 1672-1682, Jun. 2006.

[25] T. M. Tu, W. C. Cheng, C. P. Chang, P. S. Huang, and J. C. Chang, "Best tradeoff for high-resolution image fusion to preserve spatial details and minimize color distortion," IEEE Geosci. Remote Sens. Lett., vol. 4, no. 2 , pp. 302-306, Apr. 2007.

[26] J. R. Harris and R. G. Murray, "IHS transform for the integration of radar imagery with other remotely sensed data," Photogramm. Eng. Remote Sens., vol. 56, no. 12, pp. 1631-1641, Dec. 1990.

[27] T. M. Tu, P. S. Huang, C. L. Hung, and C. P. Chang, "A fast intensityhue-saturation fusion technique with spectral adjustment for IKONOS imagery," IEEE Geosci. Remote Sens. Lett., vol. 1, no. 4, pp. 309-312, Oct. 2004.

[28] R. C. Gonzalez and R. E. Woods, Digital Image Processing. Upper Saddle River, NJ, USA: Prentice-Hall, 2007.

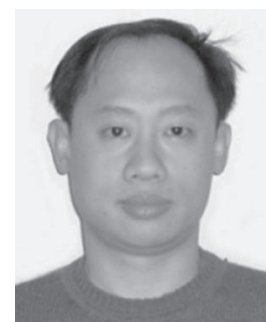

Chun-Liang Chien received the B.S. degree in biomedical engineering from Chung Yuan Christian University, Chungli, Taiwan, R.O.C., in 1998, the M.S. degree in electrical engineering from National Taiwan University, Taipei, Taiwan, in 2000, and the $\mathrm{Ph} . \mathrm{D}$. degree in computer science and information engineering from National Central University, Chungli, Taiwan, in 2011.

From 2011 to 2012, he was an Assistant Research Fellow at the Computer Vision Research Center, National Chiao Tung University, Hsinchu, Taiwan. $\mathrm{He}$ is currently an Assistant Professor of biomedical engineering, Chung Yuan Christian University. His research interests include color image processing, satellite image processing, and computer vision.

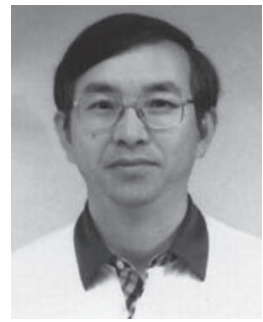

Wen-Hsiang Tsai (S'78-M'79-SM'91) received the B.S. degree from National Taiwan University, Taipei, Taiwan, in 1973, the M.S. degree from Brown University, Providence, RI, in 1977, and the Ph.D. degree from Purdue University, West Lafayette, IN, in 1979, all in electrical engineering.

Since 1979, he has been with National Chiao Tung University (NCTU), Hsinchu, Taiwan, where he is now a Chair Professor of Computer Science. At NCTU, he has served as the Head of the Department of Computer Science, the Dean of General Affairs, the Dean of Academic Affairs, and a Vice President. From 1999 to 2000, he was the Chair of the Chinese Image Processing and Pattern Recognition Society of Taiwan, and from 2004 to 2008, the Chair of the Computer Society of the IEEE Taipei Section in Taiwan. From 2004 to 2007, he was the President of Asia University, Taiwan. His current research interests include computer vision, information security, video surveillance, and autonomous vehicle applications.

Dr. Tsai has been an Editor or the Editor-in-Chief of several international journals, including Pattern Recognition, the International Journal of Pattern Recognition and Artificial Intelligence, and the Journal of Information Science and Engineering. He is currently an Editor of IEEE TRANSACTIONS ON INFORMATION FORENSICS AND SECURITY. He has published 154 journal papers and 243 conference papers, and received many awards, including the Annual Paper Award from the Pattern Recognition Society of the USA; the Academic Award of the Ministry of Education, Taiwan; the Distinguished or Outstanding Research Awards of the National Science Council, Taiwan (7 times); the ISI Citation Classic Award from Thomson Scientific, and more than 40 other academic paper awards from various academic societies. He is a Life Member of the Chinese Pattern Recognition and Image Processing Society, Taiwan. 